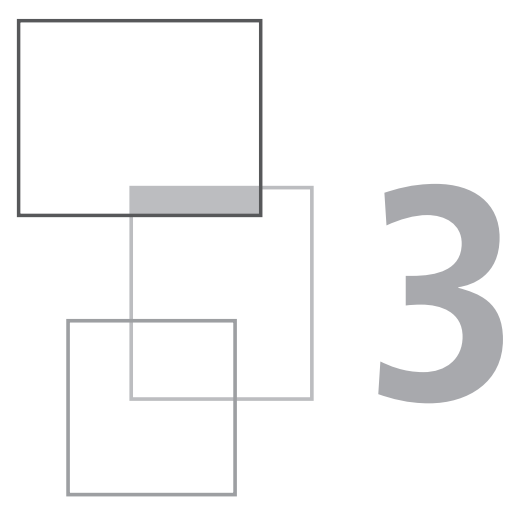

\title{
Impact sur le dérèglement climatique
}

\subsection{Le dérèglement climatique : de quoi s'agit-il ?}

Le "dérèglement climatique ", parfois appelé aussi le " changement climatique ", désigne l'ensemble des variations durables des caractéristiques climatiques en un endroit donné, à moyen ou long terme.

Le climat global de la Terre et les climats régionaux sont déterminés par le " système climatique " qui est une machine extrêmement complexe, à l'échelle de la planète. En effet, ce système est constitué de toute une série d'interactions entre différents éléments d'un ensemble constitué de cinq grandes composantes principales (catégories identifiées par Météo France) : l'atmosphère (interactions entre les vents, la composition de l'atmosphère, etc.), les surfaces continentales, l'hydrosphère (océans avec ses courants, lacs, rivières et fleuves, nappes d'eau souterraines), la cryosphère (glaces terrestres et banquise, calottes polaires, manteau neigeux) et enfin la biosphère.

Toutefois, beaucoup des phénomènes qui gouvernent ces interactions dépendent en premier lieu d'un paramètre physique fondamental qui est la température de l'atmosphère, tant par son niveau moyen régional que par sa répartition autour du globe. Pour caractériser les effets de cette température de l'atmosphère de façon aussi synthétique que possible, le plus simple est d'adopter un paramètre unique constitué par la moyenne de toutes les températures de l'atmosphère mesurées (ou calculées) autour du globe terrestre, $\mathrm{T}_{\mathrm{at}}$. Bien entendu, la connaissance de cette valeur 
ne permet pas d'en déduire le climat terrestre de façon globale, très loin s'en faut. En revanche, on sait qu'une variation de $\mathbf{T}_{\mathbf{a t}}$, même relativement minime, provoque des effets climatiques divers par le biais de tous les phénomènes que l'on vient de citer : c'est ce que l'on appelle le dérèglement climatique.

\subsection{L'effet de serre : origine et conséquences de son accroissement}

La quasi-totalité de l'énergie thermique de la Terre provient du rayonnement solaire ${ }^{13}$ dont une moitié environ parvient à traverser son atmosphère du fait de la nature de ce rayonnement (« spectre " du rayonnement solaire). Lorsque ce rayonnement vient impacter le sol, celui-ci émet un rayonnement infrarouge qui repart en direction de l'atmosphère. Mais celle-ci contient des gaz qui piègent ce type de rayonnement par leur opacité et qui en conséquence réchauffent l'atmosphère : c'est ce que l'on appelle l'effet de serre ${ }^{14}$.

Sur la Terre, le premier gaz atmosphérique naturel qui contribue à ce phénomène est la vapeur d'eau, pour $55 \%$, suivie du gaz carbonique naturel $\left(\mathbf{C O}_{2}\right)$ pour $40 \%$. Viennent ensuite, très loin derrière, le méthane et l'oxyde d'azote ainsi que l'ozone pour quelques pourcents chacun.

À cela s'ajoutent les gaz qui sont générés par les activités humaines liées essentiellement à la production d'énergie (électricité et chaleur pour usages domestiques ou industriels), aux transports (véhicules individuels, avions, navires), à l'agriculture et aux processus et produits industriels. À titre d'exemple, pour l'ensemble des 28 pays de l'Union européenne, les parts respectives de ces sources de gaz à effet de serre (GES) étaient en $2015^{15}$ de : $55 \%, 23 \%, 10 \%, 8 \%$ (au total les seules émissions de $\mathrm{CO}_{2}$ dans l'UE se sont élevées à 3,6 milliards de tonnes en 2015). Un autre exemple qu'il convient de citer est celui de la Chine puisque, en 2015, ce pays a émis plus de 10 milliards de tonnes de $\mathrm{CO}_{2}$ (essentiellement par la combustion de charbon), soit près de $30 \%$ des émissions mondiales (36 milliards de tonnes en 2015). Notons au passage que l'Union européenne émet un peu moins de $10 \%$ des

13. La Terre elle-même génère intrinsèquement de la chaleur (en partie grâce aux décroissances radioactives de radionuclides naturels tels que le thorium ou l'uranium) mais en quantités négligeables par rapport à celle apportée par le Soleil : en termes de puissance, c'est 42 TW (environ) comparé aux $170000 \mathrm{TW}$ du Soleil (soit $1370 \mathrm{~W} / \mathrm{m}^{2}$ pour des rayons perpendiculaires hors atmosphère). Notons au passage que ces chiffres peuvent être comparés à la puissance totale consommée par l'humanité qui est de 15 TW (soit en gros, 10 Mtep par an en termes d'énergie). 14. Ce phénomène physique fut identifié pour la première fois en 1824 par le savant français Joseph Fourier dans son ouvrage intitulé Mémoire sur les températures du globe terrestre et des espaces planétaires. Sans cet effet de serre, la température moyenne à la surface terrestre serait de $-18{ }^{\circ} \mathrm{C}$, alors qu'elle est en réalité de $15^{\circ} \mathrm{C}$ en moyenne grâce à cet effet de serre. Heureusement pour nous!

15. Source : Eurostat, Greenhouse gas emissions, analysis by source sector, EU-2. 
gaz à effet de serre mondiaux, moins que les États-Unis (15\%) et loin derrière la Chine $(30 \%)$. La France pour sa part n'émet qu'environ $1 \%$ du total mondial et l'Allemagne $2 \%$. Cette très bonne performance de la France en matière d'émission de $\mathrm{CO}_{2}$ s'explique essentiellement par le poids élevé du nucléaire et de l'hydraulique dans la production française d'électricité (presque $90 \%$ du mix).

Ces émissions anthropiques massives de gaz peuvent accroître notablement la concentration de certains d'entre eux dans l'atmosphère. C'est notamment le cas du $\mathrm{CO}_{2}$ généré par l'usage des combustibles fossiles (charbon, gaz, pétrole) qui contribue pour environ les deux tiers à l'augmentation artificielle de cet effet de serre. Certes, ces accroissements sont relativement faibles rapportés aux concentrations globales de gaz dans l'atmosphère, mais ils peuvent être suffisants pour engendrer par effet de serre des variations de $\mathbf{T}_{\mathbf{a t}}$ telles qu'elles peuvent perturber le climat de façon significative et durable. Ainsi, on estime actuellement que l'élévation de la température due à l'effet de serre d'origine anthropique a été de l'ordre d'un demidegré Celsius $\left({ }^{\circ} \mathrm{C}\right)$ au cours du $\mathrm{XX}^{\mathrm{e}}$ siècle, ce qui a déjà amené à des évolutions climatiques perceptibles.

Au-delà de 1 ou $2{ }^{\circ} \mathrm{C}$, il est très probable que l'on assistera à des modifications majeures du climat dont les conséquences peuvent aller jusqu'à la disparition des espèces de la faune et de la flore incapables de s'adapter rapidement. Plus généralement, ce réchauffement de l'atmosphère généralisé et assez brutal (il s’opère sur quelques dizaines d'années) fait craindre des dommages importants sur notre environnement global : élévation du niveau des mers, accentuation des événements climatiques extrêmes (sécheresses, inondations, cyclones...), déstabilisation des forêts, menaces sur les ressources d'eau douce, difficultés agricoles, désertification, réduction de la biodiversité, extension des maladies tropicales, etc.

En résumé, et pour conclure sur ce point, il est clair que la production massive d'énergie à partir de combustibles fossiles est une des principales sources d'émission de GES et que cette augmentation artificielle de GES peut conduire à des modifications significatives et durables du climat.

\subsection{Les actions internationales pour la lutte contre le dérèglement climatique}

C'est à partir de 1958 que le scientifique américain Charles Keeling commence à mesurer à Hawaï (base de Mona Loa) la concentration en $\mathrm{CO}_{2}$ dans l'atmosphère. Il en détecte des variations saisonnières mais surtout, à partir de 1961, il fournit des résultats de mesures montrant que cette concentration moyenne augmente régulièrement et de façon significative au cours du temps : c'est la courbe dite "de Keeling ». Il est alors établi rapidement que la cause première de cette augmentation est l'activité humaine, notamment via la combustion de matières fossiles.

Peu à peu la communauté internationale prend conscience de ce phénomène et de ses effets possibles sur le climat, ce qui l'amène à réunir pour la première fois 
en 1979, à Genève, une conférence mondiale sur ce sujet. Celle-ci est suivie au cours des années 1980 d'une série de conférences intergouvernementales consacrées aux changements climatiques. Dans le prolongement de ces réunions internationales, et pour faire suite à une décision du " G7 ", il est créé en novembre 1988 un groupe intergouvernemental d'experts chargé d'analyser l'évolution du climat baptisé GIEC (IPCC en anglais : Intergovernmental Panel on Climate Change). Cet organisme " a pour mission d'évaluer, sans parti-pris et de façon méthodique, claire et objective, les informations d'ordre scientifique, technique et socio-économique qui nous sont nécessaires pour mieux comprendre les risques liés au réchauffement climatique d'origine humaine, cerner plus précisément les conséquences possibles de ce changement et envisager d'éventuelles stratégies d'adaptation et d'atténuation.

Le GIEC n'a pas pour mandat d'entreprendre des travaux de recherche ni de suivre l'évolution des variables climatologiques ou d'autres paramètres pertinents. Ses évaluations sont principalement fondées sur les publications scientifiques et techniques dont la valeur scientifique est largement reconnue ». Depuis sa création, il a publié régulièrement des rapports de synthèse sur ses travaux (assessment reports) qui, à mesure de leur avancement, apportent des preuves de plus en plus probantes de la réalité du phénomène de réchauffement climatique et de son origine anthropique (même si certains opposants contestent toujours cette réalité). Globalement, il apparaît que la température moyenne de l'atmosphère terrestre a augmenté de $0,6^{\circ} \mathrm{C}$ au cours du XXe siècle et que le rythme de cette augmentation s'accélère aujourd'hui.

C'est dans le sillage de ces événements que 178 États ont adopté un premier texte officiel sur ces questions lors du premier "sommet de la Terre " qui s'est tenu à Rio de Janeiro en 1992. Il s'agit de la convention cadre des Nations unies sur les changements climatiques (CCNUCC), qui reconnaît notamment une responsabilité des pays signataires dans ce domaine. La machine est alors lancée et chaque pays signataire de la convention se doit de mettre en œuvre des solutions concrètes pour lutter contre le changement climatique, en se concertant régulièrement pour définir notamment des objectifs et prendre des engagements précis sur ces questions.

C'est l'objet des fameuses Conférences des Parties, plus connues sous leur sigle anglais COP, établies sous l'égide des Nations unies. Elles se déroulent régulièrement chaque année depuis 1995, année où elle a eu lieu la première conférence au Japon, à Kyoto (d'où résulte l'expression souvent rencontrée de " protocole de Kyoto $\left.^{16} »\right)$. Cette première conférence prévoit notamment dans un premier temps une réduction globale de GES d'au moins $5 \%$ par rapport à 1990 pour la période 2008-2012 (sauf pour les cinq pays ayant une période de référence différente).

Une seconde période d'engagement a été fixée lors du sommet de Doha en décembre 2012. Elle s'étend du $1^{\text {er }}$ janvier 2013 au 31 décembre 2020. Le « protocole " instaure en outre des obligations chiffrées de résultat pour les pays industrialisés, tout en créant pour la première fois un principe d'échange de tonnes équivalent

16. Ce protocole est officiellement entré en vigueur seulement le 16 février 2005 (après sa ratification par la Russie en octobre 2004) lors de la COP11 à Montréal, avec force de loi pour les 128 pays qui l'on ratifié. 
$\mathrm{CO}_{2}$. À noter que les sanctions consécutives au non-respect du protocole de Kyoto n'ont jamais été clairement définies en pratique et que seuls 37 pays industrialisés se sont réellement engagés sur les objectifs de ce dispositif. Cet accord n'est donc légalement pas contraignant, mais il faut souligner ici que les objectifs des pays engagés par le protocole ont toutefois été largement dépassés.

Parmi les plus marquantes de ces "COP » récentes, figure incontestablement la "COP21 » qui s'est tenue à Paris en décembre 2015 et qui a été largement médiatisée. En effet, des avancées significatives ont été enregistrées et on peut citer à ce titre le consensus obtenu sur l'objectif global qui est de contenir le réchauffement climatique en dessous de $2{ }^{\circ} \mathrm{C}$, voire de le limiter à $+1,5^{\circ} \mathrm{C}$ d'ici à la fin de ce siècle, par rapport aux températures moyennes constatées à l'ère préindustrielle. Une telle valeur peut être considérée comme un compromis politique entre ce qui semble réalisable par les différents pays et ce qui apparaît globalement tolérable du point de vue des changements climatiques à venir.

Pour atteindre cet objectif très ambitieux, tous les États se sont engagés à publier, via l'ONU, leurs propres objectifs de réduction des émissions de gaz à effet de serre (GES). Cet « accord de Paris » a été ratifié à la mi-juin 2017 par 148 des 196 parties signataires responsables à elles seules de plus de $80 \%$ des émissions de gaz à effet de serre. À noter que le retrait des États-Unis annoncé récemment par le Président américain est pour l'instant sans conséquence immédiate puisqu'il ne peut légalement prendre effet que le 4 novembre 2020, soit au lendemain de la prochaine élection présidentielle américaine.

Il reste que la mise en place d'actions concrètes s'avère complexe et plusieurs éléments risquent d'entraver sérieusement l'élan donné par la COP21, et de mettre à mal les engagements qui ont été pris à l'occasion de cet accord contraignant. Parmi ces obstacles, il faut souligner le frein au développement d'énergies décarbonées que représentent les prix particulièrement bas des énergies fossiles, et qui se maintiendront sans doute à ce faible niveau pendant de nombreuses années. En effet, les ressources associées (charbon, pétrole, gaz) demeurent abondantes et ces énergies resteront donc durablement compétitives face aux autres énergies. Par ailleurs, il est clair que certaines orientations qui visent par exemple à réduire voire à ignorer totalement l'énergie nucléaire ne favorisent pas l'atteinte des objectifs de la COP21. Pour illustrer ces difficultés au niveau européen nous reprenons ici quelques extraits de l'analyse publiée dans la dernière "Lettre de géopolitique de l'électricité ", n 76 du 30 juin 2017 :

"La Commission européenne et l'Agence européenne de l'environnement ont prévenu: l'objectif transmis par l'Union européenne aux Nations unies de réduction de $40 \%$ des émissions de gaz à effet de serre en 2030 par rapport à 1990 ne sera pas atteint avec les politiques actuelles. L'Agence de l'environnement, fin 2016, enfonce le clou : les réductions ne seraient que de 26 à $29 \%$ en 2030. Or celles-ci ont déjà diminué de $23 \%$ en 2010. Ainsi les politiques actuelles ne mèneraient qu'à des réductions supplémentaires de 3 à $6 \%$ en seize ans (2014-2030). Comment expliquer cette quasi-stagnation? Les politiques actuelles reposent, de fait, sur deux axes : l'efficacité énergétique avec mille 
milliards d'euros d'investissements en dix ans et les énergies renouvelables avec 800 milliards d'investissements déjà réalisés.

Si effectivement, les projections de l'Agence de l'environnement se confirment, la crédibilité de l'Union européenne dans sa lutte contre le réchauffement climatique sera mise à mal et son ambition de leader mondial en la matière n'aura plus de base. La Commission a proposé une nouvelle politique sur des bases inchangées : efficacité énergétique et énergies renouvelables, sans véritable marché du carbone. Les leçons de la politique précédente n'ont pas été tirées et les ratés n'ont pas été expliqués, d'où un doute quant à la pertinence des nouvelles dispositions. Il est inquiétant dans un paquet législatif intitulé "Energie propre pour tous les Européens ", que la seule mesure possible de "propreté " du mix énergétique, l'intensité des émissions par unité d'énergie consommée, ne soit pas utilisée comme donnée principale. Ceci est significatif de la réticence à créer un véritable marché du carbone».

Et l'auteur de ces lignes (Lionel Taccoen) de conclure : "Il est possible quil y ait un pilote au sein de l'Union européenne pour la lutte contre le réchauffement climatique. Pourquoi ne regarde-t-il pas son tableau de bord?". 